\title{
Classification of space-like translation surfaces in the 3-dimensional Lorentz Heisenberg group $\mathfrak{H}_{3}$
}

\author{
Rafik Medjati \\ Département de la Formation Préparatoire en Sciences et Technologies, ENP Oran, \\ Oran, Algeria, and
}

Hanifi Zoubir and Brahim Medjahdi

Ecole Nationale Polytechnique d'Oran, Oran, Algeria

\begin{abstract}
Purpose - In the Lorentz Heisenberg space $\mathrm{H} 3$ endowed with flat metric g3, a translation surface is parametrized by $r(x, y)=\gamma_{1}(x)^{*} \gamma_{2}(y)$, where $\gamma_{1}$ and $\gamma_{2}$ are two planar curves lying in planes, which are not orthogonal. In this article, we classify translation surfaces in $\mathrm{H} 3$, which satisfy some algebraic equations in terms of the coordinate functions and the Laplacian operator with respect to the first fundamental form of the surface.

Design/methodology/approach - In this paper, we classify some type of space-like translation surfaces of H3 endowed with flat metric g3 under the condition $\Delta r_{i}=\lambda_{i} r_{i}$. We will develop the system which describes surfaces of type finite in H3. For solve the system thus obtained, we will use the calculation variational. Finally, we will try to give performances geometric surfaces that meet the condition imposed.

Findings - Classification of six types of translation surfaces of finite type in the three-dimensional Lorentz Heisenberg group H3.

Originality/value - The subject of this paper lies at the border of geometry differential and spectral analysis on manifolds. Historically, the first research on the study of sub-finite type varieties began around the 1970 by B.Y.Chen. The idea was to find a better estimate of the mean total curvature of a compact subvariety of a Euclidean space. In fact, the notion of finite type subvariety is a natural extension of the notion of a minimal subvariety or surface, a notion directly linked to the calculation of variations. The goal of this work is the classification of surfaces in $\mathrm{H} 3$, in other words the surfaces which satisfy the condition/Delta (ri) = /Lambda (ri), such that the Laplacian is associated with the first, fundamental form.
\end{abstract}

Keywords Lorentz Heisenberg space, Laplace operator, Translation surface, Space-like surface

Paper type Research paper

\section{Introduction}

A Euclidean submanifold is said to be of finite Chen type if its coordinate functions are finite sum of eigenfunctions of its Laplacian [1]. Chen posed the problem of classifying the finite type surfaces in the three-dimensional Euclidean space $\mathbb{E}^{3}$. Further, the notion of finite type can be extended to any function on a submanifold of a Euclidean space or a pseudoEuclidean space.

JEL Classification - 53A10, 53C30, 53C42, 53C50

(C) Rafik Medjati, Hanifi Zoubir and Brahim Medjahdi. Published in Arab Journal of Mathematical Sciences. Published by Emerald Publishing Limited. This article is published under the Creative Commons Attribution (CC BY 4.0) licence. Anyone may reproduce, distribute, translate and create derivative works of this article (for both commercial and non-commercial purposes), subject to full attribution to the original publication and authors. The full terms of this licence may be seen at http:// creativecommons.org/licences/by/4.0/legalcode

The authors would like to thank anonymous referees for their valuable comments and careful corrections to the original version of this paper.

Received 30 March 2021 Revised 9 July 2021 20 July 2021

Accepted 24 July 2021 of translation surfaces in $\mathbb{U}_{3}$ 
A well known result, due to Takahshi [2], states that the minimal surfaces and the spheres are the only surfaces in $\mathbb{E}^{3}$ satisfying the condition.

$$
\Delta r=\lambda r, \lambda \in \mathbb{R}
$$

In [3] Ferrandez, Garay and Lucas proved that the surfaces of $\mathbb{E}^{3}$ satisfying

$$
\Delta \mathbf{H}=A \mathbf{H}, A \in M_{3}(\mathbb{R})
$$

where $\mathbf{H}$ denotes the mean curvature vector field, are either minimal, or an open piece of a sphere or of a right circular cylinder.

In [4], F. Dillen, J. Pas and L. Verstraelen proved that the only surfaces in $\mathbb{E}^{3}$ satisfying

$$
\Delta r=A r+B, A \in M_{3}(\mathbb{R}), B \in M_{3,1}(\mathbb{R})
$$

are the minimal surfaces, the spheres and the circular cylinders.

In [5], Baba-Hamed, Bekkar and Zoubir studied coordinate finite type translation surface in a three-dimensional Minkowski Space.

In [6], Yoon classified coordinate finite type translation surfaces in a three-dimensional Galilean space.

In [7], Bekkar and Senoussi studied the translation surfaces in the three-dimensional Euclidean and Lorentz-Minkowski spaces under the condition

$$
\Delta^{I I I} r_{i}=\mu_{i} r_{i}, \quad \mu_{i} \in \mathbb{R}
$$

In [8-10] Bukcu, Karacan and Yoon classified translation surfaces of type 1 and type 2 that satisfy the condition

$$
\Delta^{I, I I, I I I} x_{i}=\lambda_{i} x_{i}
$$

in the three-dimensional simply isotropic space.

Recently, in [11] Cakmak, Karakan, Kiziltug and Yoon studied the translation surfaces in three-dimensional Galilean space under the condition.

$$
\Delta^{I I} x_{i}=\lambda_{i} x_{i}
$$

In $[12,13]$ Rahmani and Rahmani has showed that modulo an automorphism of the Lie algebra, the three-dimensional Lorentz Heisenberg group $\mathbb{U}_{3}$ has the following classes of leftinvariant Lorentz metrics:

$$
\begin{aligned}
& g_{1}=-d x^{2}+d y^{2}+(x d y+d z)^{2} \\
& g_{2}=d x^{2}+d y^{2}-(x d y+d z)^{2} \\
& g_{3}=d x^{2}+(x d y+d z)^{2}-[(1-x) d y-d z]^{2} .
\end{aligned}
$$

They proved that the metrics $g_{1}, g_{2}, g_{3}$ are non-isometrics and that $g_{3}$ is flat.

Let $r: M^{2} \rightarrow \mathbb{U}_{3}$ be an isometric immersion of surface in $\mathbb{H}_{3}$. Then $r$ is said to be semiRiemannian surface in $\mathbb{H}_{3}$ if the induced metric on $M^{2}$ is non-degenerate. In that case this metric is Riemannian or Lorentzian and the surface is said to be space-like or time-like, respectively.

A space-like surface is said to be maximal if $\mathbf{H}=0$.

A time-like surface is said to be extremal surface (or minimal surface) if $\mathbf{H}=0$.

In this paper, we classify space-like translation surfaces of $\mathbb{M}_{3}$ endowed with flat metric $g_{3}$ under the condition. 


$$
\Delta r_{i}=\lambda_{i} r_{i}, \quad \lambda_{i} \in \mathbb{R}
$$

Indeed this study is closely linked to that of result, due to Dillen et al. [14] where they proved that a ruled surface $M^{2}$ in three-dimensional Minkowski space $\mathbb{R}_{1}^{3}$ is of finite type if and only if $M^{2}$ is minimal, or $M^{2}$ is a part of a circular cylinder, or $M^{2}$ is a part of a hyperbolic cylinder, or $M^{2}$ is an isoparametric surface with null rules.

\section{Preliminaries}

\subsection{The Lorentz Heisenberg group $\mathbb{M}_{3}$}

In this paragraph we shortly recall that the Heisenberg group $\mathbb{H}_{3}$ is a Lie group which is diffeomorphic to $\mathbb{R}^{3}[15]$ is defined as

$$
(x, y, z) *(\bar{x}, \bar{y}, \bar{z})=(x+\bar{x}, y+\bar{y}, z+\bar{z}-x \bar{y})
$$

The identity of the group is $(0,0,0)$ and the inverse of $(x, y, z)$ is given by $(-x,-y,-x y-z)$. The left invariant Lorentz metric on $\mathbb{H}_{3}$ is

$$
g_{3}=d x^{2}+(x d y+d z)^{2}-[(1-x) d y-d z]^{2}
$$

The following set of left-invariant vector fields forms an pseudo-orthonormal basis for corresponding Lie-algebra

$$
B=\left\{e_{1}=\frac{\partial}{\partial x}, \quad e_{2}=\frac{\partial}{\partial y}+(1-x) \frac{\partial}{\partial z}, e_{3}=\frac{\partial}{\partial y}-x \frac{\partial}{\partial z}\right\}
$$

The characterizing properties of this algebra are the following commutation relations:

$$
\left[e_{2}, e_{3}\right]=0,\left[e_{3}, e_{1}\right]=e_{2}-e_{3}, \quad\left[e_{2}, e_{1}\right]=e_{2}-e_{3} .
$$

with

$$
g_{3}\left(e_{1}, e_{1}\right)=1, g_{3}\left(e_{2}, e_{2}\right)=1, g_{3}\left(e_{3}, e_{3}\right)=-1 .
$$

If $\nabla$ is the Levi-Civita connection and $R$ is the curvature tensor of $\nabla$, we have

$$
\begin{aligned}
& \nabla_{e_{1}} e_{1}=\nabla_{e_{1}} e_{2}=\nabla_{e_{1}} e_{3}=0 \\
& \nabla_{e_{2}} e_{1}=\nabla_{e_{3}} e_{1}=e_{2}-e_{3} \\
& \nabla_{e_{2}} e_{2}=\nabla_{e_{2}} e_{3}=\nabla_{e_{3}} e_{2}=\nabla_{e_{3}} e_{3}=-e_{1} .
\end{aligned}
$$

\subsection{The Beltrami formula}

We recall that a translation surfaces $M^{2}\left(\gamma_{1}, \gamma_{2}\right)$ in the three-dimensional Lorentz Heisenberg group $\mathbb{H}_{3}$ is a surface parametrized by

$$
r: M^{2} \rightarrow \mathbb{H}_{3}, \quad r(x, y)=\gamma_{1}(x) * \gamma_{2}(y),
$$

where $\gamma_{1}$ and $\gamma_{2}$ are any generating curves in $\mathbb{R}^{3}$.

$\left(M^{2}, r\right)$ is said to be of finite Chen-type $k$ if the position vector $r$ admits the following spectral decomposition 


$$
r=r_{0}+\sum_{i=1}^{k} r_{i}
$$

where $r_{i}$ are $\mathbb{H}_{3}$-valued eigenfunctions of the Laplacian of $\left(M^{2}, r\right)$ and $\Delta r_{i}=\lambda_{i} r_{i}, \lambda_{i} \in \mathbb{R}, i=1$, $2, \ldots, k[1]$. If $\lambda_{i}$ are different, then $M^{2}$ is said to be of $k$-type.

The Laplacian $\Delta$ on $M^{2}$ is given by

$$
\Delta=\frac{-1}{\sqrt{|D|}} \sum_{i j} \frac{\partial}{\partial x^{i}}\left(\sqrt{|D|} g^{i j} \frac{\partial}{\partial x^{j}}\right) .
$$

where $G=\left(g_{i j}\right)$ is the matrix consisting of components of the induced metric on $M^{2}$, and $G^{-1}=\left(g^{i j}\right)$ is the inverse matrix of $G$ and $D=\operatorname{det} G$.

We set

$$
\Delta r=\left(\Delta r_{1}, \Delta r_{2}, \Delta r_{3}\right)
$$

such that $r=r(u, v)=\left(r_{1}(u, v), r_{2}(u, v), r_{3}(u, v)\right)$ is a function of class $C^{2}$.

We recall that the Heisenberg space endowed with a flat Lorentzian metric has the same properties as the Minkowski space, however it is well known that the position vector of a surface in a pseudo-Euclidean space is twice the mean curvature vector [1]. So we have the following result:

Theorem 1. A Beltrami formula in $\left(\mathbb{H}_{3}, g_{3}\right)$ is given by the following:

$$
\Delta r=2 \mathbf{H}
$$

where $\Delta$ is the Laplacian of the surface and $\mathbf{H}$ is the mean curvature vector field of $M^{2}$.

\section{Translation surfaces in $\left(\mathbb{U}_{3}, g_{3}\right)$}

In the Lorentz Heisenberg space $\mathbb{H}_{3}$, a translation surface is parametrized by $r(x, y)=\gamma_{1}(x) * \gamma_{2}(y)$, where $\gamma_{1}$ and $\gamma_{2}$ are two planar curves lying in planes, which are non-orthogonal and * denotes the group operation of $\mathbb{H}_{3}$.

Definition 1. A translation surface $M^{2}$ in $\mathbb{H}_{3}$ is surface parametrized by $\gamma_{1}(x) * \gamma_{2}(y)$, where $\gamma_{1}: I \subset \mathbb{R} \rightarrow \mathbb{H}_{3}, \gamma_{2}: J \subset \mathbb{R} \rightarrow \mathbb{H}_{3}$ are curves in two coordinate planes of $\mathbb{R}^{3}$.

We distinguish six types of translation surfaces in $\mathbb{H}_{3}$.

\subsection{Surfaces of type 1}

Let us first consider a translation surface $M^{2}$ parametrized by

$$
r(x, y)=(x, 0, u(x)) *(0, y, v(y))=(x, y, u(x)+v(y)-x y)
$$

where $u$ and $v$ are two smooth functions.

Thus, the basis of the tangent space $T_{p} M^{2}$ is

$$
\begin{aligned}
& r_{x}=\left(1,0, u_{x}-y\right)=\partial_{x}+\left(u_{x}-y\right) \partial_{z} \\
& r_{y}=\left(0,1, v_{y}-x\right)=\partial_{y}+\left(v_{y}-x\right) \partial_{z}
\end{aligned}
$$


That is

$$
r_{x}=e_{1}+\left(u_{x}-y\right) e_{2}-\left(u_{x}-y\right) e_{3}, r_{y}=v_{y} e_{2}+\left(1-v_{y}\right) e_{3}
$$

Therefore the coefficients of the first and second fundamental form are

$$
E=g_{3}\left(r_{x}, r_{x}\right)=1, F=g_{3}\left(r_{x}, r_{y}\right)=u_{x}-y, G=g_{3}\left(r_{y}, r_{y}\right)=2 v_{y}-1
$$

and

$$
L=g_{3}\left(\nabla_{r_{x}} r_{x}, \mathbb{N}\right), \quad M=g_{3}\left(\nabla_{r_{x}} r_{y}, \mathbb{N}\right), N=g_{3}\left(\nabla_{r_{y}} r_{y}, \mathbb{N}\right),
$$

where $\mathbb{N}$ is a unit vector field normal on $M^{2}$, which satisfies the following system

$$
\left\{\begin{array}{l}
g_{3}\left(r_{x}, \mathbb{N}\right)=0 \\
g_{3}\left(r_{y}, \mathbb{N}\right)=0 \\
g_{3}(\mathbb{N}, \mathbb{N})=-1
\end{array}\right.
$$

Classification of translation surfaces in $\mathbb{H}_{3}$

and so,

$$
\begin{aligned}
& \nabla_{r_{x}} r_{x}=u_{x x} e_{2}-u_{x x} e_{3}, \\
& \nabla_{r_{x}} r_{y}=0, \\
& \nabla_{r_{y}} r_{y}=-e_{1}+v_{y y} e_{2}-v_{y y} e_{3} .
\end{aligned}
$$

The normal unit vector field $\mathbb{N}$ to $M^{2}$ is given by:

$$
\mathbb{N}=-\frac{\left(u_{x}-y\right)}{W} e_{1}+\frac{\left(1-v_{y}\right)}{W} e_{2}+\frac{v_{y}}{W} e_{3}
$$

with

$$
W=\sqrt{E G-F^{2}}=\sqrt{2 v_{y}-\left(u_{x}-y\right)^{2}-1} .
$$

Therefore

$$
L=\frac{u_{x x}}{W}, \quad M=0, \quad N=\frac{v_{y y}+u_{x}-y}{W} .
$$

and the mean curvature vector field $\mathbf{H}$ is given by the formula:

$$
\mathbf{H}=H \mathbb{N},
$$

with

$$
H=\frac{E N+G L-2 F M}{2\left(E G-F^{2}\right)}
$$

where $H$ is, the mean curvature of the surface $M^{2}$.

So the mean curvature of the surface $M^{2}$ parametrized by $(x, y, u(x)+v(y)-x y)$ is given by

$$
H=\frac{1}{2 W^{3}}\left[v_{y y}+\left(2 v_{y}-1\right) u_{x x}+\left(u_{x}-y\right)\right]
$$


3.2 Surfaces of type 2

Now the translation surface $M^{2}$ is parametrized by

$$
r(x, y)=(0, y, v(y)) *(x, 0, u(x))=(x, y, u(x)+v(y)),
$$

where $u$ and $v$ are two smooth functions. Therefore

$$
\begin{aligned}
& r_{x}=\left(1,0, u_{x}\right)=\partial_{x}+u_{x} \partial_{z} \\
& r_{y}=\left(0,1, v_{y}\right)=\partial_{y}+v_{y} \partial_{z}
\end{aligned}
$$

and so,

$$
r_{x}=e_{1}+u_{x} e_{2}-u_{x} e_{3}, r_{y}=\left(x+v_{y}\right) e_{2}+\left(1-x-v_{y}\right) e_{3}
$$

Therefore the coefficients of the first fundamental form are

$$
E=1 \quad F=u_{x} \quad G=\left(2 v_{y}+2 x-1\right)
$$

We have

$$
\begin{aligned}
& \nabla_{r_{x}} r_{x}=u_{x x} e_{2}-u_{x x} e_{3}, \\
& \nabla_{r_{x}} r_{y}=e_{2}-e_{3}, \\
& \nabla_{r_{y}} r_{y}=-e_{1}+v_{y y} e_{2}-v_{y y} e_{3} .
\end{aligned}
$$

The normal unit vector field $\mathbb{N}$ to $M^{2}$ is given by:

$$
\mathbb{N}=-\frac{u_{x}}{W} e_{1}+\frac{\left(1-x-v_{y}\right)}{W} e_{2}+\frac{\left(x+v_{y}\right)}{W} e_{3}
$$

with

$$
W=\sqrt{E G-F^{2}}=\sqrt{2\left(v_{y}+x\right)-1-\left(u_{x}\right)^{2}} .
$$

Then the coefficients of the second fundamental form of $M^{2}$ are

$$
L=\frac{u_{x x}}{W}, \quad M=\frac{1}{W}, \quad N=\frac{u_{x}+v_{y y}}{W} .
$$

We follow the same steps as the previous type to calculate the mean curvature of the translation surface $M^{2}$. We obtain

$$
H=\frac{1}{2 W^{3}}\left[v_{y y}+\left(2 v_{y}+2 x-1\right) u_{x x}-u_{x}\right]
$$

\subsection{Surfaces of type 3}

The translation surface $M^{2}$ is parametrized by

$$
r(x, y)=(x, 0, u(x)) *(v(y), y, 0)=(x+v(y), y, u(x)-x y),
$$

where $u$ and $v$ are two smooth functions. Therefore 


$$
\begin{aligned}
& r_{x}=\left(1,0, u_{x}-y\right)=\partial_{x}+\left(u_{x}-y\right) \partial_{z} \\
& r_{y}=\left(v_{y}, 1,-x\right)=v_{y} \partial_{x}+\partial_{y}-x \partial_{z}
\end{aligned}
$$

and so,

$$
r_{x}=e_{1}+\left(u_{x}-y\right) e_{2}-\left(u_{x}-y\right) e_{3}, r_{y}=v_{y} e_{1}+e_{3},
$$

whereby the coefficients of the first fundamental form are

$$
\begin{aligned}
E=g_{3}\left(r_{x}, r_{x}\right) & =1, F=g_{3}\left(r_{x}, r_{y}\right)=u_{x}+v_{y}-y \\
G & =g_{3}\left(r_{y}, r_{y}\right)=\left(v_{y}\right)^{2}-1 .
\end{aligned}
$$

We have

$$
\begin{aligned}
& \nabla_{r_{x}} r_{x}=u_{x x} e_{2}-u_{x x} e_{3}, \\
& \nabla_{r_{x}} r_{y}=0, \\
& \nabla_{r_{y}} r_{y}=\left(v_{y y}-1\right) e_{1}+v_{y} e_{2}-v_{y} e_{3} .
\end{aligned}
$$

The normal unit vector field $\mathbb{N}$ to $M^{2}$ is given by:

$$
\mathbb{N}=\frac{1}{W}\left[\left(u_{x}-y\right) e_{1}-\left(v_{y}\left(u_{x}-y\right)+1\right) e_{2}+v_{y}\left(u_{x}-y\right) e_{3}\right]
$$

with the space-like case $W=\sqrt{-\left(u_{x}-y\right)^{2}-2 v_{y}\left(u_{x}-y\right)-1}$.

Then the coefficients of the second fundamental form of $M^{2}$ are

$$
L=-\frac{u_{x x}}{W}, \quad M=0, \quad N=\frac{\left[\left(v_{y y}-1\right)\left(u_{x}-y\right)-v_{y}\right]}{W} .
$$

We follow the same steps as the previous types to calculate the main curvature of the translation surface $M^{2}$, we obtain

$$
H=\frac{1}{2 W^{3}}\left[\left(u_{x}-y\right)\left(v_{y y}-1\right)-v_{y}-u_{x x}\left(\left(v_{y}\right)^{2}-1\right)\right]
$$

\subsection{Surfaces of type 4}

The translation surface $M^{2}$ is parametrized by

$$
r(x, y)=(v(y), y, 0) *(x, 0, u(x))=(v(y)+x, y, u(x)),
$$

where $u$ and $v$ are two smooth functions. Therefore

$$
\begin{aligned}
& r_{x}=\left(1,0, u_{x}\right)=\partial_{x}+u_{x} \partial_{z} \\
& r_{y}=\left(v_{y}, 1,0\right)=v_{y} \partial_{x}+\partial_{y}
\end{aligned}
$$


and so,

$$
r_{x}=e_{1}+u_{x} e_{2}-u_{x} e_{3}, r_{y}=v_{y} e_{1}+(v+x) e_{2}+(1-v-x) e_{3}
$$

Thus, the coefficients of the first and second fundamental form are

$$
\begin{gathered}
E=g_{3}\left(r_{x}, r_{x}\right)=1, F=g_{3}\left(r_{x}, r_{y}\right)=v_{y}+u_{x}, \\
G=g_{3}\left(r_{y}, r_{y}\right)=\left(v_{y}\right)^{2}+2 v+2 x-1 .
\end{gathered}
$$

We have

$$
\begin{aligned}
& \nabla_{r_{x}} r_{x}=u_{x x} e_{2}-u_{x x} e_{3}, \\
& \nabla_{r_{x}} r_{y}=e_{2}-e_{3}, \\
& \nabla_{r_{y}} r_{y}=\left(v_{y y}-1\right) e_{1}+2 v_{y} e_{2}-2 v_{y} e_{3} .
\end{aligned}
$$

The normal unit vector field $\mathbb{N}$ to $M^{2}$ is given by:

$$
\mathbb{N}=\frac{1}{W}\left[-u_{x} e_{1}+\left(u_{x} v_{y}+1-v-x\right) e_{2}+\left(v+x-u_{x} v_{y}\right) e_{3}\right]
$$

with the space-like case $W=\sqrt{2\left(v+x-u_{x} v_{y}\right)-\left(u_{x}\right)^{2}-1}$.

Then the coefficients of the second fundamental form of $M^{2}$ are

$$
L=\frac{u_{x x}}{W}, \quad M=\frac{1}{W}, \quad N=\frac{2 v_{y}-\left(v_{y y}-1\right) u_{x}}{W} .
$$

We follow the same steps as the previous types to calculate the main curvature of the translation surface $M^{2}$, we obtain

$$
H=\frac{1}{2 W^{3}}\left[\left(v_{y y}+1\right) u_{x}+\left[\left(v_{y}\right)^{2}+2 v+2 x-1\right] u_{x x}\right]
$$

3.5 Surfaces of type 5

In this case, the translation surface $M^{2}$ is parametrized by

$$
r(x, y)=(v(y), y, 0) *(x, 0, u(x))=(y, v(y)+x, u(x)),
$$

where $u$ and $v$ are two smooth functions. Therefore

$$
\begin{aligned}
& r_{x}=\left(0,1, u_{x}\right)=\partial_{y}+u_{x} \partial_{z} \\
& r_{y}=\left(1, v_{y}, 0\right)=\partial_{x}+v_{y} \partial_{y}
\end{aligned}
$$

and so,

$$
r_{x}=\left(y+u_{x}\right) e_{2}-\left(u_{x}+y-1\right) e_{3}, r_{y}=e_{1}+y v_{y} e_{2}+(1-y) v_{y} e_{3}
$$


Hence the coefficients of the first and second fundamental form are

$$
\begin{gathered}
E=g_{3}\left(r_{x}, r_{x}\right)=2\left(u_{x}+y\right)-1, F=g_{3}\left(r_{x}, r_{y}\right)=\left(u_{x}+2 y-1\right) v_{y}, \\
G=g_{3}\left(r_{y}, r_{y}\right)=(2 y-1)\left(v_{y}\right)^{2}+1 .
\end{gathered}
$$

We have

$$
\begin{aligned}
& \nabla_{r_{x}} r_{x}=-e_{1}+u_{x x} e_{2}-u_{x x} e_{3}, \\
& \nabla_{r_{x}} r_{y}=-v_{y} e_{1}+e_{2}-e_{3}, \\
& \nabla_{r_{y}} r_{y}=-\left(v_{y}\right)^{2} e_{1}+\left(2 v_{y}+y v_{y y}\right) e_{2}-\left(2 v_{y}+(y-1) v_{y y}\right) e_{3} .
\end{aligned}
$$

The normal unit vector field $\mathbb{N}$ to $M^{2}$ is given by:

$$
\mathbb{N}=\frac{1}{W}\left[u_{x} v_{y} e_{1}-\left(u_{x}+y-1\right) e_{2}+\left(u_{x}+y\right) e_{3}\right]
$$

with $W=\sqrt{2\left(y+u_{x}\right)-\left(u_{x}\right)^{2}\left(v_{y}\right)^{2}-1}$.

The coefficients of the second fundamental form of $M^{2}$ then are

$$
L=\frac{u_{x x}-u_{x} v_{y}}{W}, \quad M=\frac{1-u_{x}\left(v_{y}\right)^{2}}{W}, \quad N=\frac{2 v_{y}-v_{y y} u_{x}-u_{x}\left(v_{y}\right)^{3}}{W} .
$$

As in the previous types, we obtain

$$
H=\frac{1}{2 W^{3}}\left[\begin{array}{c}
{\left[(2 y-1)\left(v_{y}\right)^{2}+1\right] u_{x x}-\left[2\left(y+u_{x}\right)-1\right] v_{y y} u_{x}} \\
+\left[1-\left(2 y-1+u_{x}\right)\left(v_{y}\right)^{2}\right] u_{x} v_{y}
\end{array}\right]
$$

3.6 Surfaces of type 6

The translation surface $M^{2}$ is parametrized by

$$
r(x, y)=(y, v(y), 0) *(0, x, u(x))=(y, v(y)+x, u(x)-x y),
$$

where $u$ and $v$ are two smooth functions. Therefore

$$
r_{x}=\left(0,1, u_{x}\right)=\partial_{y}+u_{x} \partial_{z}, r_{y}=\left(1, v_{y}, 0\right)=\partial_{x}+v_{y} \partial_{y}
$$

and so,

$$
\begin{aligned}
& r_{x}=u_{x} e_{2}+\left(1-u_{x}\right) e_{3}, \\
& r_{y}=e_{1}+\left(y v_{y}-x\right) e_{2}+\left(x-y v_{y}+v_{y}\right) e_{3}
\end{aligned}
$$

Therefore the coefficients of the first and second fundamental form are

$$
\begin{gathered}
E=g_{3}\left(r_{x}, r_{x}\right)=2 u_{x}-1, F=g_{3}\left(r_{x}, r_{y}\right)=v_{y} u_{x}+y v_{y}-v_{y}-x, \\
G=g_{3}\left(r_{y}, r_{y}\right)=1-\left(v_{y}\right)^{2}+2 v_{y}\left(y v_{y}-x\right) .
\end{gathered}
$$

Classification of translation surfaces in $\mathbb{H}_{3}$ 
We have

$$
\begin{aligned}
& \nabla_{r_{x}} r_{x}=-e_{1}+u_{x x} e_{2}-u_{x x} e_{3}, \\
& \nabla_{r_{x}} r_{y}=-v_{y} e_{1}, \\
& \nabla_{r_{y}} r_{y}=-\left(v_{y}\right)^{2} e_{1}+\left(2 v_{y}+y v_{y y}\right) e_{2}-\left(2 v_{y}+y v_{y y}-v_{y y}\right) e_{3} .
\end{aligned}
$$

The normal unit vector field $\mathbb{N}$ to $M^{2}$ is given by:

$$
\mathbb{N}=\frac{1}{W}\left[\left(u_{x} v_{y}-y v_{y}+x\right) e_{1}+\left(1-u_{x}\right) e_{2}+u_{x} e_{3}\right]
$$

With $W=\sqrt{2 u_{x}-\left(u_{x} v_{y}-y v_{y}+x\right)^{2}-1}$.

Then the coefficients of the second fundamental form of $M^{2}$ are

$$
\begin{gathered}
L=\frac{u_{x x}-u_{x} v_{y}+y v_{y}-x}{W}, M=-\frac{\left(u_{x} v_{y}-y v_{y}+x\right) v_{y}}{W}, \\
N=\frac{-\left(u_{x} v_{y}-y v_{y}+x\right)\left(v_{y}\right)^{2}+2 v_{y}+y v_{y y}-v_{y y} u_{x}}{W}
\end{gathered}
$$

As above, we obtain

$$
H=\frac{1}{2 W^{3}}\left[\begin{array}{c}
\left(2 u_{x}-1\right)\left(y-u_{x}\right) v_{y y}+\left[1-\left(v_{y}\right)^{2}+2 v_{y}\left(y v_{y}-x\right)\right] u_{x x} \\
+\left(3 u_{x}+y-2\right) v_{y}-x
\end{array}\right]
$$

4. Translation surfaces in Lorentz Heisenberg space $\left(\mathbb{H}_{3}, g_{3}\right)$ satisfying $\boldsymbol{\Delta} \boldsymbol{r}_{\boldsymbol{i}}=\boldsymbol{\lambda}_{\boldsymbol{i}} \boldsymbol{r}_{\boldsymbol{i}}$ This section is devoted to classify the translation surfaces of type 1 , type 2 , type 3 , type 4 , type 5 and type 6 that satisfy the condition $\Delta r_{i}=\lambda_{i} r_{i}, \lambda_{i} \in \mathbb{R}$.

The result is:

Theorem 2. Let $M^{2}$ be a translation surface of type 1 , type 2 , type 3 , type 4 , type 5 or type 6 in the three-dimensional Lorentz Heisenberg space $\left(\mathbb{H}_{3}, g_{3}\right)$. Then $M^{2}$ satisfies the condition $\Delta r_{i}=\lambda_{i} r_{i}, i=1,2,3, \lambda_{i} \in \mathbb{R}$, if and only if $M^{2}$ has zero mean curvature.

\section{Proof of Theorem 2.}

In the following we will prove Theorem 2.

\subsection{Surfaces of type 1}

By (2.2) and (3.4), the Laplacian operator $\Delta r$ of $r$ can be expressed as

$$
\Delta r=-\frac{2\left(u_{x}-y\right)}{W} H e_{1}+\frac{2\left(1-v_{y}\right)}{W} H e_{2}+\frac{2 v_{y}}{W} H e_{3}
$$

Next, suppose $M^{2}$ satisfies condition (1.1). Then, from (3.1) and (4.1), we obtain the following system of ordinary differential equations.

$$
-\frac{2\left(u_{x}-y\right)}{W} H=\lambda_{1} x
$$




$$
\begin{aligned}
& \frac{2\left(1-v_{y}\right)}{W} H=\lambda_{2} x y+\lambda_{3}(u+v-x y) \\
& \frac{2 v_{y}}{W} H=\lambda_{2}(1-x) y-\lambda_{3}(u+v-x y)
\end{aligned}
$$

Classification of translation surfaces in $\mathbb{H}_{3}$

Therefore, the problem of classifying the translation surfaces $M^{2}$ satisfying (1.1) is reduced to the integration of the system (4.2), (4.3) and (4.4) of ordinary differential equations.

Next we study it according to the constants $\lambda_{1}, \lambda_{2}$ and $\lambda_{3}$.

Combining Equations (4.3) and (4.4) yields

$$
\begin{gathered}
-\frac{2\left(u_{x}-y\right)}{W} H=\lambda_{1} x \\
\frac{2}{W} H=\lambda_{2} x y
\end{gathered}
$$

Case 1: $\lambda_{1} \lambda_{2}=0$. Then $H=0$ and $\lambda_{3}=0$. Thus, the surface $M^{2}$ is minimal.

Case 2: $\lambda_{1} \lambda_{2} \neq 0$. Substituting (4.5) into (4.6), we get

$$
-\left(u_{x}-y\right)=\frac{\lambda_{1}}{\lambda_{2}} \frac{x}{y}
$$

Taking the partial derivative of (4.7) with respect to $x$ gives

$$
-u_{x x}=\frac{\lambda_{1}}{\lambda_{2}} \frac{1}{y}
$$

The left-hand side in (4.8) is either a constant or a function of $x$, while other side is a function of $y$. That is not possible.

\subsection{Surfaces of type 2}

We follow the same steps as the previous type. By (2.2) and (3.9), the Laplacian $\Delta r$ of $r$ can be expressed as

$$
\Delta r=-\frac{2 u_{x}}{W} H e_{1}+\frac{2\left(1-v_{y}-x\right)}{W} H e_{2}+\frac{2\left(v_{y}+x\right)}{W} H e_{3}
$$

Suppose that $M^{2}$ satisfies the condition (1.1). Then, from (3.6) and (4.9), we obtain the following system of ordinary differential equations.

$$
\begin{gathered}
-\frac{2 u_{x}}{W} H=\lambda_{1} x \\
\frac{2\left(1-v_{y}-x\right)}{W} H=\lambda_{2} x y+\lambda_{3}(u+v) \\
\frac{2\left(v_{y}+x\right)}{W} H=\lambda_{2}(1-x) y-\lambda_{3}(u+v)
\end{gathered}
$$

Therefore, the problem of classifying the translation surfaces $M^{2}$ satisfying (1.1) is reduced to the integration of the system (4.10), (4.11) and (4.12) of ordinary differential equations. 
Applying similar algebraic methods, used in the case of surface of type 1 , we will study this system according to the values of the constants $\lambda_{1}, \lambda_{2}, \lambda_{3}$.

By combining Equations (4.11) and (4.12) we obtain

$$
\begin{gathered}
-\frac{2 u_{x}}{W} H=\lambda_{1} x \\
\frac{2}{W} H=\lambda_{2} y
\end{gathered}
$$

Case 1: $\lambda_{1} \lambda_{2}=0$.

(1) If $\lambda_{2}=0$, then we have $H=0$. Thus, the surface $M^{2}$ is minimal.

(2) If $\lambda_{2} \neq 0$, from (4.13), we obtain $u_{x}=0$. Substituting (3.10) into (4.14), with $W=\sqrt{2\left(v_{y}+x\right)-1-\left(u_{x}\right)^{2}}$, we get

$$
v_{y y}=\lambda_{2} y\left[2\left(v_{y}+x\right)-1\right]^{2}
$$

Taking the partial derivative of (4.15) with respect to $x$ gives

$$
v_{y}=\frac{1-2 x}{2}
$$

The left-hand side in (4.16) is either a constant or a function of $y$, whereas other side is a function of $x$. That is not possible.

Case 2: $\lambda_{1} \lambda_{2} \neq 0$. Substituting (4.13) into (4.14), we get

$$
-u_{x}=\frac{\lambda_{1}}{\lambda_{2}} \frac{x}{y}
$$

Taking the partial derivative of (4.17) with respect to $x$ gives

$$
-u_{x x}=\frac{\lambda_{1}}{\lambda_{2}} \frac{1}{y}
$$

The left-hand side in (4.18) is a constant or a function of $x$, whilst other side is a function of $y$. That is not possible.

4.3 Surfaces of type 3

By following the same steps as the previous types, by (2.2) and (3.14), we obtain

$$
\Delta r=\frac{2\left(u_{x}-y\right)}{W} H e_{1}-\frac{2\left[v_{y}\left(u_{x}-y\right)+1\right]}{W} H e_{2}+\frac{2 v_{y}\left(u_{x}-y\right)}{W} H e_{3}
$$

Suppose that $M^{2}$ satisfies condition (1.1). Then, from (3.11) and (4.19), we obtain the following system of ordinary differential equations.

$$
\begin{gathered}
\frac{2\left(u_{x}-y\right)}{W} H=\lambda_{1}(x+v) \\
-\frac{2\left[v_{y}\left(u_{x}-y\right)+1\right]}{W} H=\lambda_{2}(x+v) y+\lambda_{3}(u-x y)
\end{gathered}
$$




$$
\frac{2 v_{y}\left(u_{x}-y\right)}{W} H=(1-(x+v)) \lambda_{2} y-\lambda_{3}(u-x y)
$$

Combining Equations (4.21) and (4.22) we have

$$
\begin{aligned}
\frac{2\left(u_{x}-y\right)}{W} H & =\lambda_{1}(x+v) \\
-\frac{2}{W} H & =\lambda_{2} y
\end{aligned}
$$

Case 1: $\lambda_{1} \lambda_{2}=0$. Then $H=0$ and $\lambda_{3}=0$. Thus, the surface $M^{2}$ is minimal.

Case 2: $\lambda_{1} \lambda_{2} \neq 0$. Substituting (4.23) into (4.24), we get

$$
-\left(u_{x}-y\right)=\frac{\lambda_{1}}{\lambda_{2}} \frac{(x+v)}{y}
$$

Taking the partial derivative of (4.25) with respect to $x$ gives

$$
-u_{x x}=\frac{\lambda_{1}}{\lambda_{2}} \frac{1}{y}
$$

We have an identity of two functions, one depending only on $x$ and the other one depending only on $y$. That is not possible.

\subsection{Surfaces of type 4}

By (2.2) and (3.19), we obtain

$$
\Delta r=-\frac{2 u_{x}}{W} H e_{1}+\frac{2\left(u_{x} v_{y}+1-v-x\right)}{W} H e_{2}+\frac{2\left(v+x-u_{x} v_{y}\right)}{W} H e_{3}
$$

Suppose that $M^{2}$ satisfies the condition (1.1). Then, from (3.16) and (4.27), we obtain the following system of ordinary differential equations.

$$
\begin{gathered}
-\frac{2 u_{x}}{W} H=\lambda_{1}(v+x) \\
\frac{2\left(u_{x} v_{y}+1-v-x\right)}{W} H=\lambda_{2}(v+x) y+\lambda_{3} u \\
\frac{2\left(v+x-u_{x} v_{y}\right)}{W} H=\lambda_{2}\left((1-(v+x)) y-\lambda_{3} u\right)
\end{gathered}
$$

Combining Equations (4.29) and (4.30) we have

$$
\begin{gathered}
-\frac{2 u_{x}}{W} H=\lambda_{1}(v+x) \\
\frac{2}{W} H=\lambda_{2} y
\end{gathered}
$$

Case 1: $\lambda_{1} \lambda_{2}=0$.

(1) If $\lambda_{2}=0$, then we have $H=0$. Thus, the surface $M^{2}$ is minimal.

(2) If $\lambda_{2} \neq 0$, from (4.31), we obtain $u_{x}=0$. Then from (3.20) we have $H=0$ and $\lambda_{2}=0$, which is contradiction. 
Case 2: $\lambda_{1} \lambda_{2} \neq 0$. Substituting (4.31) into (4.32), we get

$$
-u_{x}=\frac{\lambda_{1}}{\lambda_{2}} \frac{(v+x)}{y}
$$

Taking the partial derivative of (4.33) with respect to $x$ gives

$$
-u_{x x}=\frac{\lambda_{1}}{\lambda_{2}} \frac{1}{y}
$$

We have an identity of two functions, one depending only on $x$ and the other one depending only on $y$. That is impossible.

4.5 Surfaces of type 5

By (2.2) and (3.24), the Laplacian $\Delta$ of $r$ can be expressed as

$$
\Delta r=\frac{2 u_{x} v_{y}}{W} H e_{1}-\frac{2\left(u_{x}+y-1\right)}{W} H e_{2}+\frac{2\left(u_{x}+y\right)}{W} H e_{3}
$$

Suppose that $M^{2}$ satisfies condition (1.1). Then, from (3.21) and (4.35), we obtain the following system of ordinary differential equations.

$$
\begin{gathered}
\frac{2 u_{x} v_{y}}{W} H=\lambda_{1} y \\
-\frac{2\left(u_{x}+y-1\right)}{W} H=\lambda_{2} y(v+x)+\lambda_{3} u \\
\frac{2\left(u_{x}+y\right)}{W} H=\lambda_{2}(1-y)(v+x)-\lambda_{3} u
\end{gathered}
$$

Combining the Equations (4.37) and (4.38) we have

$$
\begin{gathered}
\frac{2 u_{x} v_{y}}{W} H=\lambda_{1} y \\
\frac{2}{W} H=\lambda_{2}(v+x) \\
\frac{2\left(u_{x}+2 y-1\right)}{W} H=-\lambda_{3} u
\end{gathered}
$$

Case 1: $\lambda_{3}=0$. Since $u$ depends only on $x$, then from (4.41) $H=0$ and $\lambda_{1}=\lambda_{2}=\lambda_{3}=0$. Thus, the surface $M^{2}$ is minimal.

Case 2: $\lambda_{3} \neq 0$.

(1) If $\lambda_{2}=0$, from (4.40) $H=0$, and $\lambda_{3}=0$, which is contradiction.

(2) If $\lambda_{1}=0$ and $\lambda_{2} \neq 0$ from (4.39) we obtain $u_{x} v_{y}=0$. Then

- If $u_{x}=0$. From (3.25) we obtain $H=0$ and $\lambda_{3}=0$, which is a contradiction. 
- If $v_{y}=0$, then $v$ is constant $(v=a)$. By combining Equations (3.25) and (4.40) and the fact that $W=\sqrt{2\left(y+u_{x}\right)-\left(u_{x}\right)^{2}\left(v_{y}\right)^{2}-1}$, we conclude

$$
u_{x x}=\lambda_{2}(a+x)\left[2 y+2 u_{x}-1\right]^{2}
$$

Taking the partial derivative of (4.42) with respect to $y$ gives

$$
u_{x}=\frac{1-2 y}{2}
$$

The right-hand side in (4.43) is either a function of $y$ while other side is a constant or a function of $x$. That is impossible.

(3) If $\lambda_{1} \lambda_{2} \neq 0$. Substituting (4.39) into (4.40) and (4.41) into (4.40) we get

$$
\begin{gathered}
u_{x} v_{y}=\frac{\lambda_{1}}{\lambda_{2}} \frac{y}{(v+x)} \\
\left(u_{x}+2 y-1\right)=-\frac{\lambda_{3}}{\lambda_{2}} \frac{u}{(v+x)}
\end{gathered}
$$

Combining Equations (4.44) and (4.45) we have

$$
\lambda_{2}(2 y-1)(v+x) v_{y}=\lambda_{3} u v_{y}+\lambda_{1} y
$$

Taking the partial derivative of (4.46) with respect to $x$ and the fact that $v_{y} \neq 0$, we obtain

$$
u_{x}=\frac{\lambda_{2}}{\lambda_{3}}(2 y-1)
$$

That is impossible because (4.47) is an identity of two functions, one depending only on $x$ and the other one depending only on $y$.

\subsection{Surfaces of type 6}

By (2.2) and (3.29), we have

$$
\Delta r=\frac{2\left(u_{x} v_{y}-y v_{y}+x\right)}{W} H e_{1}+\frac{2\left(1-u_{x}\right)}{W} H e_{2}+\frac{2 u_{x}}{W} H e_{3}
$$

Suppose that $M^{2}$ satisfies condition (1.1). Then, from (3.26) and (4.48), we obtain the following system of ordinary differential equations.

$$
\begin{gathered}
\frac{2\left(u_{x} v_{y}-y v_{y}+x\right)}{W} H=\lambda_{1} y \\
\frac{2\left(1-u_{x}\right)}{W} H=\lambda_{2} y(v+x)+\lambda_{3}(u-x y) \\
\frac{2\left(x+u_{x}-y\right)}{W} H=\lambda_{2}(1-y)(v+x)-\lambda_{3}(u-x y)
\end{gathered}
$$

Classification of translation surfaces in $\mathbb{H}_{3}$ 
Combining the Equations (4.50) and (4.51) we have

$$
\begin{gathered}
\frac{2\left(u_{x} v_{y}-y v_{y}+x\right)}{W} H=\lambda_{1} y \\
\frac{2(x-y+1)}{W} H=\lambda_{2}(v+x) \\
\frac{2\left[1-u_{x}-(x-y+1) y\right]}{W} H=\lambda_{3}(u-x y)
\end{gathered}
$$

Case 1: $\lambda_{3}=0$ and $H \neq 0$. Then from (4.54) we obtain

$$
1-u_{x}-(x-y+1) y=0
$$

Taking the partial derivative of (4.55) with respect to $x$, we obtain

$$
u_{x x}=y
$$

That is impossible because $u_{x x}$ is function of $x$. Then $H=0$ and $\lambda_{1}=\lambda_{2}=\lambda_{3}=0$. Thus, the surface $M^{2}$ is minimal.

Case 2: $\lambda_{3} \neq 0$.

(1) If $\lambda_{1} \lambda_{2}=0$. from (4.52), we have

$$
u_{x} v_{y}-y v_{y}+x=0
$$

Taking the partial derivative of (4.57) with respect to $x$, we obtain

$$
u_{x x}=-\frac{1}{v_{y}}
$$

Then both sides have to equal a nonzero constant, namely

$$
u_{x x}=a=-\frac{1}{v_{y}}
$$

Which implies that $v_{y}=-\frac{1}{a}$. Considering it into (4.57) gives

$$
-\frac{u_{x}}{a}+x=-\frac{y}{a}
$$

The left-hand side in (4.59) is either constant or a function of $x$ while other side is a function of $y$. That is impossible.

(2) If $\lambda_{1} \lambda_{2} \neq 0$. Substituting (4.53) into (4.54), we get

$$
\left[1-u_{x}-(x-y+1) y\right](v+x)=\frac{\lambda_{3}}{\lambda_{2}}(u-x y)(x-y+1)
$$

The partial derivative of (4.60) with respect to $x$ and $y$ yields

$$
2 x-2 y+1+v-\left(u_{x x}-y\right) v_{y}=\frac{\lambda_{3}}{\lambda_{2}}\left(2 x-2 y+1+u_{x}\right)
$$


Taking partial derivative of (4.61) with respect to $x$ and $y$ leads to

$$
u_{x x x} v_{y y}=0
$$

We have two situations:

(1) $u_{x x x}=0$ i.e.

$$
u_{x}=b_{1} x+b_{2}
$$

Then (4.61) reduces to

$$
2 x+1-\frac{\lambda_{3}}{\lambda_{2}}\left(2 x+1+b_{1} x+b_{2}\right)=\left(b_{1}-y\right) v_{y}-v+2 y\left(1-\frac{\lambda_{3}}{\lambda_{2}}\right)
$$

We have an identity of two functions, one depending only on $x$ and the other one depending only on $y$. That is impossible.

(2) $v_{y y}=0$, i.e.

$$
v=c_{1} y+c_{2} .
$$

Then (4.61) reduces to

$$
2 x-u_{x x} c_{1}-\frac{\lambda_{3}}{\lambda_{2}}\left(2 x+1+u_{x}\right)+c_{2}+1=2\left(1-c_{1}-\frac{\lambda_{3}}{\lambda_{2}}\right) y
$$

The left-hand side in (4.63) is either constant or a function of $x$ while other side is a function of $y$. That is impossible.

\section{Conclusion}

In this work, we give another characterization of translation surfaces in the Lorentz Heisenberg space $\mathbb{H}_{3}$ equipped with a flat metric. This is done by showing that these translation surfaces satisfy Condition $\Delta r_{i}=\lambda_{i} r_{i}, i=1,2,3, \lambda_{i} \in \mathbb{R}$, if and only if they have zero mean curvature.

\section{References}

1. Chen BY., Total mean curvature and submanifolds of finite type, Singapore; World Scientific: 1984.

2. Takahashi T, Minimal immersions of Riemannian manifolds, J Math Soc Japan. 1966; 18: 380-85.

3. Ferrandez A, Garay OJ, Lucas P, On a certain class of conformally flat Euclidean hypersurfaces, Proceeding of the Conference Global Analysis and Global Differential Geometry, Berlin: 1990.

4. Dillen F, Pas J, Verstraelen L. On surfaces of finite type in Euclidean 3-space, Kodai Math J. 1990: $10-21$.

5. Baba-Hamed C, Bekkar M, Zoubir H, Translation surfaces in the three-dimensional Lorentz Minkowski space satisfying $\Delta r_{i}=\lambda_{i} r_{i}$, Int J Math Anal. 2010; 4(17): 797-808.

6. Yoon DW, Some classification of translation surfaces in Galilean 3-space, Int J Math Anal. 2012; 6(28): $1355-61$.

7. Bekkar M, Senoussi B, Translation surfaces in the 3-dimensional space satisfying $\Delta^{I I I} r_{i}=\mu_{i} r_{i}$ J Geom. 2012; 103: 367-74.

8. Bukcu B, Karacan MK, Yoon DW, Translation surfaces of type 2 in the three dimensional simply isotropic space $\square_{3}^{1}$, Bull Korean Math Soc. 2017; 54(3): 953-65. 
9. Bukcu B, Yoon DW, Karacan MK, Translation surfaces in the three dimensional simply isotropic space $\square_{3}^{1}$ satisfying $\Delta^{I I I} x_{i}=\lambda_{i} x_{i}$, Konuralp J Math. 2016; 4(1): 275-81.

10. Karacan MK, Yoon DW, Bukcu B, Translation surfaces in the three dimensional simply isotropic space $\rrbracket_{3}^{1}$, Int J Geomet Methods Mod Phys. 2016; 13: 9.

11. Cakmak A, Karakan MK, Kiziltug S, Yoon DW, Translation surfaces in the three dimensional Galilean space satisfying $\Delta^{I I} x_{i}=\lambda_{i} x_{i}$, Bull Korean Math Soc. 2017; 54(4): 1241-54.

12. Rahmani N, Rahmani S, Lorentzian geometry of the Heisenberg group, Geom Dedicata. 2006; 118: $133-40$.

13. Rahmani N, Rahmani S, Structures Homogenes Lorentziennes sur le Groupe de Heisenberg, J Geom Phys. 1994; 13: 254-58.

14. Dillen F, Van De Woestyne I, Verstraelen L, Walrave J. Ruled surfaces of finite type in 3dimensional Minkowski space, Results Math. 1995; 27: 250-55.

15. Batat W, Rahmani S, Isometries, Geodesics and Jacobi fields of Lorentzian Heisenberg group, Mediterr J Math. 2011; 8: 411-30.

\section{Corresponding author}

Rafik Medjati can be contacted at: rafik.medjati@enp-oran.dz

For instructions on how to order reprints of this article, please visit our website: 